Kamila StAUdigl-CieCHOWICZ, Wien

\title{
Das Kriegswirtschaftliche Ermächtigungsgesetz 1917 Von seiner Entstehung 1917 bis zu seiner Aufhebung 1946
}

\author{
KwEG 1917-1946. From the genesis to the abolition \\ of the Austrian Wartime Economy Enabling Act \\ In 1917, the Austrian parliament passed a statute - entitled 'Kriegswirtschaftliches Ermächtigungsgesetz' (KwEG) \\ - that empowered the government to enact statutory orders on matters concerning wartime economy. The article \\ describes the genesis of the KwEG and discusses the use of this emergency tool in interwar Austria. Although the \\ war ended in 1918, the government continued to pass ordinances based on the KwEG until 1934. This was possible \\ due to the vague wording of the provisions of this law. The article illustrates the different opinions on the use and \\ abuse of this statute.
}

Keywords: Austrian constitutional history - emergency regulation interwar period - statutory orders - wartime economy

\section{Entstehungsgeschichte}

Die Idee des Kriegswirtschaftlichen Ermächtigungsgesetzes 1917 (KwEG) geht zurück auf eine 1914 ergangene kaiserliche Notverordnung gem. § 14 Dezemberverfassung 1867. Während das § 14-Notverordnungsrecht dem Kaiser weitreichende Befugnisse gab, ermächtigte eine darauf basierende kaiserliche Verordnung die Regierung im eingeschränkten Bereich der wirtschaftlichen Kriegsmaßnahmen Verordnungen zu erlassen. ${ }^{1}$ Erst mit der formellen Gesetzwerdung 1917 wurde der Ermächtigungsbereich explizit erweitert, bis er über staatsrechtliche Brüche hinaus im Interpretationsweg gar als Legitimierung zur Erlassung einer neuen Verfassung 1934 herangezogen wurde. Doch die Anfänge dieses Gesetzes waren zunächst noch vergleichsweise klein.

\footnotetext{
${ }^{1}$ Siehe dazu den Beitrag von Anita Ziegerhofer in diesem Band.
}

\subsection{Die KwEVO als "sekundäres Notverordnungsrecht ${ }^{\text {" }_{2}}$}

Anfang Oktober 1914 erließ der Kaiser unter Gegenzeichnung des Gesamtministeriums eine Verordnung, welche der Regierung weitreichende Ermächtigungen im Bereich der Kriegswirtschaft gab. ${ }^{3}$ Als Grundlage diente ihm das $\S 14$ Notverordnungsrecht der Dezemberverfassung 1867.4 Die Regierung wurde darin "ermächtigt, durch Verordnung die notwendigen Verfügungen zur Förderung des wirtschaftlichen Lebens, insbesondere der Landwirtschaft, der Industrie, des Handels und Gewerbes, fer-

\footnotetext{
${ }^{2}$ WITTMAYER, Notverordnungsrechte 43.

${ }^{3}$ RGBl. 274/1917. In weiterer Folge als KwEVO bezeichnet. Vgl. dazu auch MERKL, Verordnungsgewalt $398 f$.

${ }^{4}$ Siehe dazu die Beiträge von Christian NESCHWARA und von Helmut GEBHARDT in diesem Band. Vgl. auch HASIBA, Notverordnungsrecht.
} 
ner zur Approvisionierung der Bevölkerung zu treffen. ${ }^{\circ} 5$

Anders als das $\S 14$ Notverordnungsrecht sah die Verordnung für die auf ihr basierenden Verordnungen keine Vorlagepflicht nach Einberufung des Reichsrates vor. Allerdings waren entsprechende Verordnungen „nach Wiedereintritt normaler Zustände sofort außer Kraft zu setzen."6 Als Begründung für die Erlassung der KwEVO wurde 1917 anlässlich der Vorlage der Notverordnungen an das Abgeordnetenhaus in aller Kürze festgehalten: „Infolge der kriegerischen Ereignisse machte die Gestaltung des wirtschaftlichen Lebens eine Reihe von Maßnahmen im Interesse der rationellen Verwertung und ökonomischen Ausnützung der reichen natürlichen Hilfsmittel sowie zur Abwehr schädlicher Einflüsse auf wirtschaftlichem Gebiete notwendig. Für diese zum Schutze der durch den Krieg gefährdeten öffentlichen Interessen auf den verschiedenen Wirtschaftsgebieten unentbehrlich erscheinenden Maßnahmen wurde in der vorliegenden Kaiserlichen Verordnung die erforderliche gesetzliche Grundlage geschaffen. ${ }^{\text {" }}$ Die knappe Begründung zeigt ganz klar, dass die ursprüngliche Ermächtigungsverordnung dezidiert für rein wirtschaftliche Zwecke gedacht war.

\subsection{Einberufung des Reichsrates}

Bereits im Sommer 1916 gab es - allerdings erfolglose - Bestrebungen den Reichsrat einzuberufen. Erst im Mai 1917 unter der Regierung Clam-Martinic wurde der Reichsrat zum ersten Mal nach über drei Jahren einberufen. ${ }^{8}$ Ein zentrales Thema der folgenden Sitzungen waren die gem. § 14 erlassenen kaiserlichen Notverordnungen. Neben dem Verfassungsausschuss be-

\footnotetext{
5 Art. 1 KwEVO.

${ }^{6}$ Art. 3 KwEVO.

755 BglAH 12. GP XXII. Sess., 3.

${ }^{8}$ HASIBA, Kriegswirtschaftliches Ermächtigungsgesetz 549.
}

schäftigte sich primär ein eigener Kriegswirtschaftlicher Ausschuss mit den zwischen 1914 und 1917 ergangenen Not- bzw. Regierungsverordnungen. ${ }^{9}$ In der Praxis diente die KwEVO der Erlassung „sämtliche[r] wirtschaftliche[r] Kriegsmaßnahmen mit Ausnahme jener finanzieller Natur". ${ }^{10}$ Zwischen Oktober 1914 und Juli 1917 ergingen über 500 Verordnungen, die sich auf die KwEVO beriefen. ${ }^{11}$

\subsection{Kriegswirtschaftlicher Ausschuss}

Gleich nach Einberufung des Reichsrates Ende Mai 1917 wurde die KwEVO dem Abgeordnetenhaus vorgelegt. Mitte Juni 1917 erfolgte die Zuweisung an den Kriegswirtschaftlichen Ausschuss, drei Wochen später hatte dieser seinen Bericht erstellt. Im Kriegswirtschaftlichen Ausschuss war man sich unsicher, wie dieses „Unikum" zu behandeln war. ${ }^{12}$ Der Bericht verwies darauf, dass „die Anwendung des § 14 zu einem Ermächtigungsgesetz" staatsrechtlich ein „Unikum" sei und bemerkte, dass sie auch faktisch „von einer ungeheuren, kaum übersehbaren Tragweite" war, folglich das Parlament gefordert sei, „einen verfassungsmäßigen Rechtsboden " herzustellen. ${ }^{13}$

Eine konkrete Zusammenstellung der Verordnungen lag dem Ausschuss nicht vor, so dass selbst die genaue Zahl der auf Grund der KwEVO erlassenen Verordnungen dem Ausschuss nicht bekannt war. Klar war, dass es sich "um viele Hunderte solcher Verordnungen“ handelte. „Nahezu die ganze Lebensmittel- und Rohstoffwirtschaft, die Einrichtung des Generalkommissariats für die Übergangswirtschaft, verschiedene Kredit- und Gebührenverordnungen, kurz der weitaus überwiegende Teil des

\footnotetext{
${ }^{9}$ HASIBA, Kriegswirtschaftliches Ermächtigungsgesetz 551.

${ }^{10}$ MERKL, Verordnungsgewalt 398.

${ }^{11} \mathrm{Vgl}$. dazu näher in diesem Band Anita ZIEGERHOFER.

12 AB 429 BlgAH 12. GP XXII. Sess., 1.

${ }^{13}$ AB 429 BlgAH 12. GP XXII. Sess., 1.
} 
gesamten kriegswirtschaftlichen Systems, ruht auf jener Rahmenverordnung. " ${ }^{14}$ Der Ausschuss zog verschiedene Lösungsansätze in Betracht. Große Sorge bereitete ihm der Umstand, dass „die meisten der abgeleiteten Ministerialverordnungen [...] wieder Ermächtigungen und Aufträge für die politischen Behörden 2. und 1. Instanz [enthielten] und [...] sich somit in unübersehbarer Ausdehnung über das ganze wirtschaftliche Leben bis in seine feinsten Ausläufer verästelt" haben. ${ }^{15}$ Die Beibehaltung der KwEVO schien untragbar, aber bei deren Aufhebung drohte Rechtschaos.

Der Ausschussbericht erläuterte die Konsequenzen beider Lösungen: Für den Fall, dass der Reichsrat die KwEVO unbehandelt bestehen ließe, hatte das entweder eine faktisch oder eine rechtlich problematische Situation zur Folge. Klar war, "daß bereits in der nächsten Zeit neuerlich sehr eingreifende kriegswirtschaftliche Verfügungen unerläßlich notwendig“ sein würden. Behandelte der Reichsrat die KwEVO nun nicht, so „würde nämlich das Haus entweder den noch nicht dagewesenen Zustand bewirken, daß die Gesetzgebung und der $\S 14$ nebeneinander und gleichzeitig arbeiten, oder es würde die Verantwortung dafür tragen, daß jede Fortführung der Kriegswirtschaft zunächst unterbleibt. ${ }^{" 16}$ Da das Anwenden des $\S 14$ während der Reichsrat aktiv tagte rechtliche Fragen aufwerfen würde, konnte die vorübergehende Einstellung der Verordnungspraxis nach der KwEVO der Kriegswirtschaft Schäden zufügen. Darüber hinaus hätte das Hinwegsehen über die KwEVO dem Prestige des Reichsrats geschadet, insbesondere da gerade der Reichsrat die $\S 14$ Praxis öffentlich beklagte.

In Frage kam anderseits die gänzliche Aufhebung der KwEVO. Doch auch dieser Weg erschien dem Ausschuss nicht ratsam. Einerseits

\footnotetext{
${ }^{14}$ AB 429 BlgAH 12. GP XXII. Sess., 1.

${ }^{15}$ AB 429 BlgAH 12. GP XXII. Sess., 1.

${ }^{16}$ AB 429 BlgAH 12. GP XXII. Sess., 2.
}

sprach dagegen - wie auch schon bei der ersten Lösung - die Befürchtung, dass „ein vollständiges Stocken der weiteren Kriegswirtschaft ${ }^{\prime \prime 17}$ folgen würde. Andererseits schreckte der Ausschuss vor dieser Lösung zurück, da sie einen rechtlichen Rattenschwanz von unabsehbarem Ausmaß nach sich ziehen würde. Innerhalb der drei Jahre waren Ermächtigungen nach der KwEVO in manche Bereiche der Kriegswirtschaft bis ins Innerste durchgedrungen, so dass die Aufhebung der Rahmenverordnung ein "katastrophales Rechtschaos" erwarten ließ.18 Der Ausschuss sträubte sich vor diesem Schritt, der „den bekannten großen Rechtsstreit wieder aufrollen [würde], welche Rechtswirkung für die Vergangenheit daraus entstehen. Bleiben falls die Kaiserliche Verordnung aufgehoben wird - die von ihr abgeleiteten Verordnungen und Verfügungen der Regierung in Kraft oder nicht?" ${ }^{\prime 19}$

Als Ausweg aus dieser schwierigen Situation schlug der Kriegswirtschaftliche Ausschuss schließlich die Erlassung eines Kriegswirtschaftlichen Ermächtigungsgesetzes, „welches für die Zukunft und Vergangenheit klare, verfassungsmäßige Zustände herstellt und mit seinem Inkrafttreten, also ohne jedes Vakuum, die Kaiserliche Verordnung vom 10. Oktober 1914 aufhebt", vor. ${ }^{20}$

\section{Das KwEG $1917^{21}$}

Gleichzeitig mit der Ablehnung der weiteren Auseinandersetzung mit der KwEVO brachte der Kriegswirtschaftliche Ausschuss einen Ge-

\footnotetext{
${ }_{17}$ AB 429 BlgAH 12. GP XXII. Sess., 2.

18 AB 429 BlgAH 12. GP XXII. Sess., 2.

${ }^{19}$ AB 429 BlgAH 12. GP XXII. Sess., 2.

${ }^{20}$ AB 429 BlgAH 12. GP XXII. Sess., 2.

${ }^{21}$ Vgl. zum KwEG: HUEMER, Hecht 138-156; HASIBA, Kriegswirtschaftliches Ermächtigungsgesetz; LEIDINGER, MORITZ, Kriegswirtschaftliches Ermächtigungsgesetz.
} 
setzesentwurf zur Sprache, der die KwEVO ersetzen sollte. Das Gesetz orientierte sich zwar an der aufzuhebenden Verordnung, dehnte aber den Ermächtigungsbereich darüber hinaus aus.

\subsection{Die Vorbilder}

Als Vorbilder fungierten die KwEVO, aber auch das deutsche Ermächtigungsgesetz. ${ }^{22}$ Im Deutschen Reich wurde ein auf den Krieg ausgerichtetes Ermächtigungsgesetz bereits Anfang August 1914 erlassen. ${ }^{23}$ Darin wurde der Bundesrat ermächtigt die gesetzlichen Maßnahmen anzuordnen, „,welche sich zur ,Abhilfe wirtschaftlicher Schädigungen' als notwendig" erwiesen.24 Im Ausschuss entfachten Diskussionen über die Ausgestaltung des Ermächtigungsrahmens. Während manche Mitglieder eine möglichst enge Fassung befürworteten, forderten andere „eine Vorkehrung, die das Entstehen neuer Kriegszentralen verhindert oder Schädigungen der Produktion ausschließt und dergleichen mehr." 25 Schlussendlich war dem Ausschuss sowohl die in der KwEVO normierte „Förderung des wirtschaftlichen Lebens", als auch die "Abhilfe wirtschaftlicher Schäden" nach dem deutschen Ermächtigungsgesetz zu wenig, so dass darüber hinaus der "ganze Komplex der sogenannten Übergangswirtschaft" dem sachlichen Ermächtigungsbereich beigefügt wurde. Gerechtfertigt wurde der breite Ermächtigungsrahmen mit dem Umstand, dass „sich die vielgestaltigen Aufgaben der kommenden Kriegswirtschaft keineswegs mit jener Sicherheit übersehen [ließen], welche eine inhaltliche Einschränkung der Ermächtigung rechtfertigen würde". ${ }^{26}$ Trotzdem wollte man keinen Freibrief für die Regierung schaffen, zunächst gab es die

\footnotetext{
${ }^{22}$ AB 429 BlgAH 12. GP XXII. Sess., 2.

${ }^{23} \mathrm{Vgl}$. dazu in diesem Band Christoph GusY; WITT-

MAYER, Notverordnungsrechte 36.

${ }^{24}$ AB 429 BlgAH 12. GP XXII. Sess., 2.

${ }^{25}$ AB 429 BlgAH 12. GP XXII. Sess., 2.

${ }^{26}$ AB 429 BlgAH 12. GP XXII. Sess., 2.
}

Überlegung, die betreffenden Verordnungen an die Zustimmung bzw. zumindest Anhörung des kriegswirtschaftlichen Ausschusses zu binden. Diese Idee wurde schließlich aufgegeben, der Ausschuss wollte „die hier versuchte Verwischung der Grenzen zwischen Legislative und Exekutive" vermeiden und die Rechtssetzungsprozesse kurz und einfach gestalten. ${ }^{27}$

\subsection{Das KwEG im Plenum}

Der Gesetzesentwurf wurde am 10. Juli 1917 im Plenum des Abgeordnetenhauses behandelt. Für den Kriegswirtschaftlichen Ausschuss berichtete der Jurist und Abgeordnete (Deutscher Nationalverband) Robert Freißler. Er plädierte dafür, die KwEVO als „Monstrosität in unserem Verfassungsleben" $28 \mathrm{zu}$ beseitigen und die künftige kriegswirtschaftliche Ordnung auf gesetzliche Basis zu setzen. Besonders betonte er die Notwendigkeit, rasch und flexibel kriegswirtschaftliche Anordnungen zu verfügen. Das KwEG sollte für die Regierung als flexibles Werkzeug dienen, jedoch, gleichzeitig durch die Vorlagepflicht der Verordnungen, dem Parlament ein Kontrollrecht sichern. In der Debatte wurde mit Nachdruck betont, dass die Zustimmung zum KwEG keinesfalls als Vertrauensvotum für die Regierung und die bisherige kriegswirtschaftliche Praxis zu verstehen sei. Verschiedene Vorschläge der Beschränkung der Regierung in ihrer Verordnungsgewalt konnten sich nicht durchsetzen. Unter anderem war eine Beschränkung der Ermächtigung auf vier Monate oder eine monatliche Vorlagepflicht der Regierung gefordert worden. Zustimmung hingegen bekam der Änderungsantrag, der die Meinungsäußerung eines Ausschusses des Abgeordnetenhauses vor der Erlassung von Verordnungen allgemeiner Art vorsah. ${ }^{29}$

\footnotetext{
${ }^{27}$ AB 429 BlgAH 12. GP XXII. Sess., 3.

28 Sten. Prot AH 12. GP XXII. Sess., 812.

${ }^{29}$ HASIBA, Kriegswirtschaftliches Ermächtigungsgesetz 555 .
} 
Insgesamt drehte sich die Diskussion primär um die Missstände in der Kriegswirtschaft. Ein dominierendes Thema war der unzureichende Schutz der Landbevölkerung, der Arbeiter und der Kleingewerbetreibender. Insbesondere die Verordnungen zu den Höchstpreisen und die Requisition von Getreide wurden auf das Heftigste kritisiert. Kaum problematisiert wurde die Frage der Reichweite der kriegswirtschaftlichen Regierungsverordnungen. Der christlich-soziale Abgeordnete Matthäus Bauchinger definierte die der Regierung übertragenen Vollmachten als Rechte, "die beinahe an gesetzgeberische Rechte streifen" ${ }^{30}$ doch der Frage, wie weit die Ermächtigung reiche, ging man nicht nach.

Das Herrenhaus sprach sich gegen den vom Abgeordnetenhaus geforderten Ausschuss aus. ${ }^{31}$ Schlussendlich nahm das Abgeordnetenhaus die vom Herrenhaus vorgeschlagene Version des Gesetzes am 14. Juli 1917 an. Lediglich eine Resolution wurde beigefügt. Darin wurde die Regierung aufgefordert, „vor Erlassung kriegswirtschaftlicher Verordnungen von grundsätzlicher Wichtigkeit nach Tunlichkeit mit dem Kriegswirtschaftlichen-, beziehungsweise dem Ernährungsausschusse des Abgeordnetenhauses Fühlung zu nehmen." ${ }^{\prime 32}$ Am 24. Juli erhielt das Gesetz die kaiserliche Sanktion, drei Tage später wurde es im Reichsgesetzblatt verlautbart.

\subsection{Vorlagepflicht}

Typisch für Notverordnungen war deren Vorlagepflicht im Parlament, ein Schutzmechanismus, der vor Missbrauch des Notverordnungsrechts schützen sollte. Nun sah $\S 14$ zwar eine Vorlagepflicht vor, nicht jedoch die KwEVO. Das führte zu der paradoxen Situation, dass zwar

\footnotetext{
${ }^{30}$ Sten. Prot AH 12. GP XXII. Sess., 821.

${ }^{31}$ HAsIBA, Kriegswirtschaftliches Ermächtigungsgesetz 555 .

32 Schreiben an das Herrenhaus v. 14. 7. 1917, Parlamentsarchiv Wien, Bestand Herrenhaus, 42 (H 3c-3-14).
}

kaiserliche Verordnungen nach $\S 14$ dem versammelten Reichsrat vorgelegt werden mussten, nicht jedoch die auf der Rahmenverordnung ergangenen Regierungsverordnungen. Um dieser Situation Herr zu werden, sah das KwEG 1917 nun einen Passus vor, der sich mit den auf der KwEVO basierenden Regierungsverordnungen beschäftigte. Demnach war gem. §3 KwEG die Regierung verpflichtet, alle auf Grund der KwEVO „erlassenen Verordnungen dem Reichsrat vorzulegen und über sein Verlangen außer Wirksamkeit zu setzen. “33 Dabei wurde bewusst die Formulierung ,außer Wirksamkeit zu setzen“, verwendet; eine Formulierung, die vom $\S 14$ StGG entlehnt worden war. Damit wollte man klarstellen, dass die Aufhebung ex nunc erfolgte - eine Frage, die für $§ 14$ von Theorie und Judikatur bereits einhellig beantwortet worden war. ${ }^{34}$ Anders als bei Gesetzen, bedurfte es für die Aufhebung einer Regierungsverordnung lediglich gleichlautender Beschlüsse beider Häuser, worauf die Regierung tätig werden musste. Der kriegswirtschaftliche Ausschuss betonte, dass dadurch „der Einfluß der Regierung und die Aufstellung von Sanktionshindernissen" ausgeschaltet wurde. ${ }^{35}$ Die Vorlagepflicht war ein wichtiges Kontrollrecht des Parlaments über die Kriegswirtschaft und die gesetzeskonforme Anwendung durch die Regierung. Für den Fall der Nichteinhaltung der Vorlagepflicht erklärte der Ausschuss, dass es sich um "Zwangstermine“ handle, deren Missachtung ein automatisches Außer-WirksamkeitTreten der Verordnungen zur Folge hätte. ${ }^{36}$

Auf diese nachträgliche Sanierung durch das KwEG nahm auch der VfGH Bezug. Im Dezember 1919 beschäftigte er sich im Rahmen einer

\footnotetext{
${ }^{33} \S 3$ KwEG.

${ }^{34}$ AB 429 BlgAH 12. GP XXII. Sess., 4.

${ }^{35}$ AB 429 BlgAH 12. GP XXII. Sess., 4.

${ }^{36}$ AB 429 BlgAH 12. GP XXII. Sess., 4. Auffallend ist, dass diese Rechtsansicht im 1920 verfassten Kommentar zum B-VG gar nicht erwähnt wird.
} 
Beschwerde wegen der Verletzung des Grundrechts auf den gesetzlichen Richter mit einem 1916 durch Verordnung des Handelsministers geschaffenen Schiedsgericht. ${ }^{37}$ Die Verordnung berief sich auf die KwEVO. Der VfGH erklärte in den Entscheidungsgründen, dass es nicht notwendig sei zu prüfen, ob „,bei dieser Rechtslage die Einsetzung des Schiedsgerichtes mit dem ihm in der Verordnung gegebenen Wirkungskreise in rechtsgültiger Weise erfolgen konnte", da die Verordnung und ihr Inhalt ,ihre sichere Grundlage nachträglich durch das" KwEG erlangt hätten. ${ }^{38}$

Mit der Rezeption der gesamten Rechtsordnung von der Monarchie zur Republik 1918 kam die Frage auf, inwiefern die Vorlagepflicht bestand, wo das Organ "Reichsrat" nicht mehr vorhanden war. Der Kommentar zum VerfassungsÜbergangsgesetz 1920 erklärte, dass keine rechtliche Verpflichtung zur Vorlage der Regierungsverordnungen mehr bestehe. Als Begründung führte er an, dass bereits nach dem Staatsgründungsbeschluss diese Pflicht weggefallen sei, „da der Reichsrat keine in die Republik übernommene Institution“39 sei. In der Praxis wurden die Regierungsverordnungen dem Nationalrat vorgelegt. Unterließ die Regierung die Vorlage, so hatte das auf die Gültigkeit der Regierungsverordnung keinerlei Einfluss, wobei diese Rechtsansicht - so der Kommentar - bereits zu monarchischen Zeiten vertreten wurde. Auffallend ist dabei die Diskrepanz zum Ausschussbericht zum KwEG aus 1917, hieß es dort doch, dass die Vorlagetermine Zwangstermine seien und "bei Nichteinhaltung die Verordnungen automatisch außer Wirksamkeit treten". ${ }^{40}$ Das Gesetz selbst erklärte im § 5 Abs. 2 KwEG, dass die auf dem KwEG basierenden Verordnungen „außer Wirksamkeit zu setzen [sind],

\footnotetext{
${ }^{37}$ Erk. v. 17. 12. 1919, Zl. 333.

${ }^{38}$ Erk. v. 17. 12. 1919, Zl. 333.

${ }^{39}$ Kelsen, Froehlich, MerKL, Bundesverfassung 311.

${ }^{40}$ AB 429 BlgAH 12. GP XXII. Sess., 4.
}

wenn sie dem Reichsrat zu dem im §3 [KwEG] bezeichneten Termin nicht vorgelegt werden."

Der wesentliche Unterschied zeigt sich lediglich bei der Frage der Ministerverantwortlichkeit: Während das Unterlassen der Vorlage in der Monarchie „unter der Sanktion der Ministerverantwortlichkeit stand", 41 konnte eine solche wohl nun angesichts der mangelnden rechtlichen Verpflichtung nicht drohen. Allerdings zeigt ein Blick auf die Judikatur des Verfassungsgerichtshofes, dass auch diese Frage keineswegs klar war. 1924 stellte der VfGH in seinen Entscheidungsgründen fest, dass die Unterlassung der Vorlage nicht die Folge habe, „daß die betreffende Verordnung von selbst außer Kraft tritt; sie macht nur die Regierung eventuell verantwortlich. ${ }^{42}$ Eindeutiger hatte sich der VfGH noch zwei Jahre zuvor zu dieser Frage ausgesprochen, in dem er den Rechtssatz aufstellte, dass die Unterlassung der Vorlage „lediglich die zur Vorlage verpflichteten Minister verantwortlich" mache.43

\section{Der Ermächtigungsbereich}

\subsection{Der Begriff „Regierung" - persönlicher Ermächtigungsbereich}

$\S 1$ KwEG ermächtigte die Regierung zur Erlassung entsprechender Verordnungen. Unter dem Begriff „Regierung" wurden in der Monarchie sowohl die Gesamtregierung als auch die einzelnen Staatsminister verstanden. Deutlich stellte dies 1919 der VfGH fest. In einem Erkenntnis vom Dezember 1919 beschäftigte er sich mit der Frage des personellen Ermächtigungsrahmens. ${ }^{44}$ Entgegen der Meinung der Salzburger Landes-

\footnotetext{
${ }^{41}$ Kelsen, Froehlich, MerKL, Bundesverfassung 311.

${ }^{42}$ Erk. v. 15. 12. 1924, Zl. V 6/24. Hervorhebung durch

Autorin vorgenommen.

${ }^{43}$ Erk. v. 21. 6. 1922, Zl. V 6/22.

${ }^{44}$ Erk. v. 16. 12. 1919, Zl. 331.
} 
regierung erklärte der $\mathrm{VfGH}$, dass unter dem in $\S 1$ KwEG verwendeten Begriff "Regierung“ stets die "Staatsregierung" zu verstehen sei. In den Entscheidungsgründen leitete der VfGH seine Rechtsansicht mittels historischer und systematischer Interpretation, so wie der Auslegung des Wortlauts her. Demnach ermächtigte das KwEG nur die "Staatsregierung“ bzw. ihre Mitglieder, nicht jedoch die „Landesregierung“. Mit $\S 7$ Verfassungs-Übergangsgesetz 1920, der sich mit dem Übergang der Zuständigkeiten in das vom B-VG 1920 geschaffene System beschäftigte, wurde die Zuständigkeit, Verordnungen nach dem KwEG 1917 zu erlassen, explizit sowohl der Bundesregierung als auch den einzelnen Bundesministern zugesprochen. Der Kommentar zum B-VG 1920, der auch das Verfassungs-Übergangsgesetz umfasste, sah die „ausdrückliche Delegierung auch der einzelnen Bundesminister [...] darum geboten, weil unter ,Bundesregierung' nur das Kollegium, nicht aber auch die einzelnen Minister zu verstehen sind, während unter ,Regierung' im Sinne des kriegswirtschaftlichen Ermächtigungsgesetzes sowohl das Gesamtministerium als auch die einzelnen Minister zu verstehen waren. ${ }^{\text {“45 Näher }}$ konkretisiert wurde diese Frage 1921 durch den Verfassungsgerichtshof, der grundsätzlich feststellte, dass unter „Regierung“ „die Staatsbehörden zu verstehen [seien], die innerhalb ihres amtlichen Wirkungskreises Verordnungen $\mathrm{zu}$ erlassen befugt sind, insbesondere also die einzelnen Minister innerhalb ihres amtlichen Wirkungskreises. ${ }^{46}$

\subsection{Der zeitliche Ermächtigungsbereich}

Bereits bei den Verhandlungen zum KwEG 1917 im Kriegswirtschaftlichen Ausschuss wurde der Gedanke einer zeitlichen Befristung der Ermächtigung erörtert. ${ }^{47}$ Der Antrag, , die Ermächtigung

\footnotetext{
45 Kelsen, Froehlich, MerKL, Bundesverfassung 311.

${ }^{46}$ Erk. v. 24. 1. 1921, Zl. D 2/20.

${ }^{47}$ AB 429 BlgAH 12. GP XXII. Sess., 3.
}

nur auf vier Monate zu erteilen", ${ }^{48}$ wurde jedoch abgelehnt. Die Begründung für die Ablehnung der Befristung liest sich wie ein Treppenwitz der Geschichte - man sah in einem befristeten Gesetz "für das Parlament, besonders in den gegenwärtigen Zeiten, eine gewisse Gefahr ${ }^{\prime 49}$. Konkret wurde befürchtet, dass durch die Auslastung des Reichsrates eine Verlängerung der Befristung verabsäumt werden könnte, was zu Schäden in der Kriegswirtschaft führen würde. Künftige Schwierigkeiten, das Gesetz wieder loszuwerden, wurden nicht vorhergesehen. Im Gegenteil, mit einem unbefristeten Gesetz sah sich der Ausschuss auf der sicheren Seite: „,... so steht es dem Parlament jederzeit frei, nach seinem eigenen Ermessen und ohne den Druck von Zwangsmaßnahmen die Materie neuerlich in Verhandlung zu ziehen und das Gesetz je nach der Lage der Verhältnisse abzuändern oder unter Umständen ganz aufzuheben." ${ }^{\text {50 }}$

Die Frage nach der Dauer der Anwendbarkeit des KwEG 1917 stellte sich im Jänner 1921 der VfGH bei der Prüfung der Gültigkeit einer 1918 erlassenen Vollzugsanweisung. In den Entscheidungsgründen erklärte der VfGH, dass mit „der Beendigung des Krieges [...] die durch den Krieg hervorgerufenen außerordentlichen Verhältnisse noch kein Ende erreicht [haben]. Die Erfahrung, die jedermann im täglichen Leben macht, zeigt, daß diese außerordentlichen Verhältnisse noch heute fortdauern. Die Umwälzungen im ganzen wirtschaftlichen Leben sind bis zum heutigen Tage zu keinem Abschluß gelangt, so daß außer allen Zweifel zur Zeit des Erlassens der Vollzugsanweisung [...] 1918 durch den Krieg hervorgerufene außerordentliche Verhältnisse bestanden." 51 Darüber hinaus verwies der VfGH auf das Verfassungs-Übergangsgesetz aus 1920 und die damit eingeführte Notwendigkeit, per

\footnotetext{
${ }^{48}$ AB 429 BlgAH 12. GP XXII. Sess., 3.

${ }^{49}$ AB 429 BlgAH 12. GP XXII. Sess., 3.

${ }^{50}$ AB 429 BlgAH 12. GP XXII. Sess., 3.

${ }^{51}$ Erk. v. 24. 1. 1921, Zl. D 2/20.
} 
Bundesgesetz die Behebung der außerordentlichen Verhältnisse festzustellen. Da bis 1921 ein solches Bundesgesetz nicht ergangen war, bedeutete das im Umkehrschluss, dass die außerordentlichen Verhältnisse andauerten. ${ }^{52}$ Mehrere Anläufe seitens der Sozialdemokraten, ein entsprechendes Gesetz in den 1920er und 1930er Jahren durchzusetzen, blieben erfolglos. ${ }^{53}$

\subsection{Sachlicher Ermächtigungsbereich}

Der sachliche Ermächtigungsbereich für die Erlassung von Regierungsverordnungen ergibt sich klar aus dem Gesetzestext. Gem. § 1 Abs. 1 KwEG wurde die Regierung ermächtigt „die notwendigen Verfügungen zur Förderung und Wiederaufrichtung des wirtschaftlichen Lebens, zur Abwehr wirtschaftlicher Schädigungen und zur Versorgung der Bevölkerung mit Nahrungsmitteln und anderen Bedarfsgegenständen zu treffen."54 Der Ermächtigungsrahmen war bewusst breiter gefasst, um auf die unvorhersehbaren Entwicklungen in der Kriegswirtschaft und der Übergangswirtschaft vorbereitet $\mathrm{zu}$ sein. ${ }^{55}$

Die enumerative Aufzählung der Gebiete, auf die sich die Regierungsverordnungen erstrecken konnten, verhinderte freilich nicht Diskussionen über die Zulässigkeit einzelner Regierungsverordnungen. Die Interpretation der verwendeten Begriffe konnte je nach politischer Zielsetzung und Rechtsanwendungsposition unterschiedlich ausfallen. In den 1920er Jahren beschäftigte sich der Verfassungsgerichtshof vereinzelt mit der Frage, ob der sachliche Ermächtigungsbereich des KwEG überschritten wurde. ${ }^{56}$ Dabei stellte er fest, dass Verordnungen, die darauf abstellten, „dem allgemeinen wirtschaftlichen Wohle widersprechende Spekulationsgeschäfte zu ver-

\footnotetext{
${ }^{52}$ Erk. v. 24. 1. 1921, Zl. D 2/20.

${ }_{53}$ Vgl. dazu unten Punkt 5.2.

${ }^{54} \S 1$ Abs. 1 KwEG.

${ }^{55}$ Vgl. bereits oben Punkt 2.1.

${ }^{56}$ Erk. v. 24. 1. 1921, Zl. D 2/20.
}

hindern" als „Wirtschaft fördernd“ in den Ermächtigungsbereich fallen. ${ }^{57}$ Auch die „Erhaltung der Demobilisierungsgüter" wurde als eine „Maßnahme zur Abwehr wirtschaftlicher Schäden"58 bewertet. Ausführlich mit dem sachlichen Ermächtigungsbereich des KwEG beschäftigte sich angesichts der Fülle neuer Regierungsverordnungen 1933 der Staatsrechtler Max Layer in einem kritischen Beitrag. Der Rechtsansicht Adolf Merkls ${ }^{59}$ folgend erklärte Layer jene Materien, die vom Notverordnungsrecht des Bundespräsidenten ausgenommen waren, $^{60}$ nun auch für KwEG-Verordnungen unantastbar. Merkl und Layer begründeten dies mit dem Argumentum a maiori ad minus ${ }^{61}$ - „es ist unmöglich, daß der Gesetzgeber von 1929 diese Materien selbst den gesetzändernden Verordnungen des B.-Präsidenten [sic] gegenüber unbedingt der Gesetzgebung vorbehalten wollte, nicht aber gegenüber den einfachen Verordnungen auf Grund des Ermächtigungsgesetzes."62 Auch die leeren Floskeln der austrofaschistischen Regierung, die jegliche Materien unter den Anwendungsbereich subsumierte, kritisierte Layer pointiert: „Niemand wird behaupten können, daß die gänzliche oder teilweise Lahmlegung des Verfassungsgerichtshofes zur Förderung des wirtschaftlichen Lebens und Versorgung der Bevölkerung mit Nahrungsmitteln erforderlich gewesen sei." 63 Freilich änderte dies nichts am weiteren Vorgehen der autoritären Regierung, die das KwEG gar benutzte, um 1934 eine neue Verfassung $\mathrm{zu}$ erlassen. Das stellte einen offensichtlichen Verfassungsbruch dar,

\footnotetext{
${ }^{57}$ Erk. v. 24. 1. 1921, Zl. D 2/20.

${ }^{58}$ Erk. v. 24. 1. 1921, Zl. D 2/20.

${ }^{59}$ MERKL, Verordnungsvollmacht.

${ }^{60}$ Zum Notverordnungsrecht des Bundespräsidenten vgl. den Beitrag von Ewald WIEDERIN in diesem Band.

${ }^{61}$ Anders sieht das HoRN, Gesetzgeber 234.

${ }^{62}$ LAYER, Ermächtigungsbereich 213.

${ }^{63}$ LAYER, Ermächtigungsbereich 218. Zur Ausschaltung des VfGH vgl. den Beitrag von Stephan HINGHOFER-SZALKAY in diesem Band.
} 
unterlag angesichts des ausgeschalteten Verfassungsgerichtshofes jedoch keiner formalen Kontrolle.

\subsection{Der Strafrahmen in den Verordnungen}

Die Regierung war befugt Strafbestimmungen in den nach der KwEVO bzw. dem KwEG erlassenen Regierungsverordnungen vorzusehen. Während die KwEVO als Höchststrafe eine Geldstrafe von $5000 \mathrm{~K}$ oder eine maximal sechsmonatige Freiheitsstrafe normierte, wurden die potenziellen Strafbestimmungen durch das KwEG verschärft. § 2 KwEG ermächtigte die Regierung in den Regierungsverordnungen „für Übertretungen Geldstrafen bis zu 20.000 K, Arreststrafen bis zu sechs Monaten, de[n] Verfall von Gegenständen, auf die sich die strafbare Handlung [bezog], und de[n] Verlust von Gewerbeberechtigungen“ festzusetzen. Die Erhöhung des Strafrahmens und Erweiterung der Strafarten war notwendig gewesen, da die „Geldstrafen bis zu $5000 \mathrm{~K}$ sehr häufig gänzlich wirkungslos“ waren, „weil der durch die Übertretung kriegswirtschaftlicher Vorschriften erzielbare Gewinn ein vielfaches dieser Summe" betrug. ${ }^{64}$ Hinzu kam, dass die unterschiedlichen Strafen nun nicht mehr alternativ, sondern kumulativ verhängt werden konnten.

Bei den Beratungen des Kriegswirtschaftlichen Ausschusses zum KwEG war erwogen worden, noch höhere Geld- und Freiheitsstrafen zu ermöglichen. Man löste sich jedoch von diesem Gedanken, sollte doch diese Strafgewalt bei den politischen Behörden bleiben. Da „das administrative Strafrecht kein streng geregeltes und umschriebenes Verfahren“ hatte, musste "eine gewisse Mäßigung bei der Festsetzung der Obergrenze eingehalten werden. ${ }^{\prime 65}$ Manche Mitglieder des Ausschusses hingegen sahen die Mög-

\footnotetext{
${ }^{64}$ AB 429 BlgAH 12. GP XXII. Sess., 3.

${ }^{65}$ AB 429 BlgAH 12. GP XXII. Sess., 3.
}

lichkeit der Verhängung von Freiheitsstrafen durch die politischen Behörden als problematisch an. Der Abgeordnete Cäsar Haller v. Hallenburg, Mitglied des Polenklubs, stellte einen Minoritätsantrag, der eine „Verdopplung der Zuständigkeit" vorsah: Während für Freiheitsstrafen die Gerichte zuständig wären, fielen alle anderen Strafen den politischen Behörden zu. ${ }^{66}$ Dieser konnte sich nicht durchsetzen.

\subsection{Wirkung der KwEG-Verordnungen}

Weder aus den parlamentarischen Materialien, noch aus dem Gesetzestext selbst ergibt sich die Reichweite der KwEG-Verordnungen. Folglich wurde die Frage, ob KwEG-Verordnungen gesetzesändernde Kraft zukam, immer wieder behandelt - mit unterschiedlichem Ergebnis. Kurz nach dem Zustandekommen des KwEG äußerte sich der Staatsrechtler Leo Wittmayer zu dieser Frage. Für ihn waren KwEG-Verordnungen nicht mit Notverordnungen gleichzusetzen, er erblickte in ihnen eine "leichtere Ware“ als in den "sakralen Notverordnungen“.67 Wittmayer qualifizierte KwEG-Verordnungen als eine res sui generis, eine "aus dem Rahmen der Verfassung völlig heraustretende Eigen$\operatorname{art}^{\prime \prime}{ }^{68}$ Demnach sollten diese Verordnungen offenbar ,in ihrer staatsrechtlichen Energie zwischen den banalen Ausführungsrechtsverordnungen zu bestimmten Gesetzen und den feierlichen Notverordnungen, von denen sie sich bloß gradweise unterscheiden, die richtige Mitte halten ". ${ }^{69}$ Wittmayer zweifelte an einer allfälligen gesetzesändernden Wirkung der KwEGVerordnungen. Als Hauptargument für seine Zweifel nannte er den Umstand, dass das KwEG-Verordnungsrecht im Gegensatz zum Notverordnungsrecht auch neben einem ver-

\footnotetext{
66429 BlgAH 12. GP XXII. Sess., 9.

${ }^{67}$ WITTMAYER, Ermächtigungsgesetz 365.

${ }^{68} \mathrm{Ebd}$.

${ }^{69}$ Ebd. 366.
} 
sammelten Reichsrat ausgeübt werden konnte. ${ }^{70}$ Anders beantwortete die Frage nach der Wirkung der KwEG-Verordnungen der Verfassungsgerichtshof. 1922 erklärte dieser mit Berufung auf frühere Judikatur, dass das KwEG „der Staatsregierung so weitgehende Vollmachten gegeben [habe], daß sie auch Abänderungen von Bestimmungen des Staatsgrundgesetzes treffen kann". ${ }^{71}$ Ausführlicher ging der VfGH Ende 1924 auf diese Problematik ein. ${ }^{72}$ Zwar erkannte er, dass eine "solche Möglichkeit [...] vom Standpunkte der Idee des Rechtsstaates bedenklich erscheinen [mag], allein das wirtschaftliche Ermächtigungsgesetz ist auch in der neuen Bundesverfassung rezipiert worden und steht so lange in Geltung, bis es ausdrücklich aufgehoben wird."73 Folglich stellte er den Rechtssatz auf, dass das KwEG "die Regierung auch zur Erlassung gesetzändernder Verordnungen“ delegiere. ${ }^{74}$ Auf diese Entscheidungen beriefen sich regierungsnahe Juristen, wie beispielsweise Robert Hecht, in ihren Begründungen der rechtmäßigen Vorgehensweise der Verordnungspraxis im Austrofaschismus.75 Ganz anders beurteilte diese Frage Max Layer 1933. In seinem kritischen Aufsatz zum KwEG erklärte er, dass so „allgemeine und weitgehende Übertragungen der Befugnis allgemeine Normen zu erlassen" zunächst einmal eines Verfassungsgesetzes und nicht nur eines einfachen Gesetzes bedurft hätten. Zwar war dieser Mangel für Layer durch die Übernahme des KwEG im Verfassungs-Übergangsgesetz 1920 saniert worden, ${ }^{76}$ doch änderte das nichts daran, dass eine ausdrückliche Anordnung der gesetzändernden

\footnotetext{
${ }^{70}$ Ebd. 364f.

${ }^{71}$ Erk. v. 15. 12. 1922, Zl. V 22/22.

${ }^{72}$ Erk. v. 15. 12. 1924, Zl. V 6/24; vgl. dazu auch den Beitrag von Ilse REITER-ZATLOUKAL in diesem Band.

${ }^{73}$ Erk. v. 15. 12. 1924, Zl. V 6/24.

${ }^{74}$ Erk. v. 15. 12. 1924, Zl. V 6/24.

${ }^{75}$ HUEMER, Sektionschef Hecht 154.

${ }^{76}$ Vgl. dazu weiter unten Punkt 5.1, sowie der Beitrag von Ilse REITER-ZATLOUKAL in diesem Band.
}

Wirkung von KwEG-Verordnungen fehlte, folglich nur Rechtsverordnungen praeter legem erlassen werden durften. ${ }^{77}$ Maßgeblich war für Layer dabei die Frage, ob eine inhaltliche Veränderung eines Gesetzes durch die Regierungsverordnung erfolgt war. Beschränkte sich eine KwEG-Verordnung darauf, die Geltungsdauer eines Gesetzes zu verlängern oder „die Anwendung einzelner Bestimmungen auf begrenzte Zeit“ zu hemmen, so lag streng "genommen [...] ja auch in diesen Verordnungen ein Eingriff in die Gesetzgebung, aber da eine inhaltliche Veränderung des Gesetzes nicht bewirkt wird, könnte man solche Verordnungen durch das kriegsw. Ermächtigungsgesetz als ausreichend gedeckt ansehen. " 78 Adolf Merkl ging zwar von einer gesetzändernden Wirkung der KwEGVerordnungen aus, mahnte aber im Frühjahr 1933 bei der Auslegung der Schranken der Verordnungsvollmacht eine restriktive Vorgehensweise ein. ${ }^{79}$ Er betonte, dass gerade die Ermächtigung zu gesetzändernden Verordnungen keinesfalls extensiv interpretiert werden dürfe, da dies dem bundestaatlichen Prinzip und dem Legalitätsprinzip widersprechen würde. Eine klare Verletzung der Schranken der Verordnungsvollmacht sah Merkl in der Erlassung der Verfassung 1934 mittels KwEG-Verordnung, denn eine verfassungsändernde Kraft hatte das KwEG nicht - weder in der Monarchie noch in der Republik. Scharfsinnig konstatierte Merkl, dass die offizielle Annahme der verfassungsändernden Kraft der KwEG-Verordnungen der Idee der Monarchie widersprach: „Die Ermächtigung der Regierung oder einzelner Minister zu verfassungsändernden Verordnungen hätte nämlich diesen eine größere Macht eingeräumt, als selbst dem Kaiser zustand. Im Falle der verfassungsändernden Kraft der kriegswirtschaftlichen Verordnungen hätte ja die Regierung oder

\footnotetext{
77 LAYER, Ermächtigungsbereich 206f.

${ }^{78}$ LAYER, Ermächtigungsbereich 210.

${ }^{79}$ MERKL, Verordnungsvollmacht 137.
} 
sogar ein einzelner Minister nach freiem Ermessen die Monarchie aufheben können. Will man wirklich ernstlich behaupten, daß Kaiser und Reichsrat einem Vollzugsorgan mit dem kriegswirtschaftlichen Ermächtigungsgesetz die Vollmacht erteilt haben, sie selbst in legitimer Weise zu depossedieren?!“80

\section{Regierungsverordnungen 1917/18}

Bis zum Ende der Monarchie wurden insgesamt 304 Regierungsverordnungen nach dem KwEG erlassen. ${ }^{81} \mathrm{Um}$ die Dimensionen dieses Gesetzes $\mathrm{zu}$ erfassen, muss die Anzahl der KwEGVerordnungen freilich im Gesamtkontext der ordentlichen und außerordentlichen Gesetzgebung betrachtet werden. Das Reichsgesetzblatt verzeichnete in der zweiten Hälfte des Jahres 1917, gerechnet $a b$ der Kundmachung des KwEG, 218 Verlautbarungen. Davon entfielen 115, also 53 Prozent aller Verlautbarungen ab dem 27. Juli 1917, auf Regierungsverordnungen nach dem KwEG. Zwar verzeichnete das Reichsgesetzblatt im Jahr 1918 sogar 189 Regierungsverordnungen nach dem KwEG, doch waren diese über einen breiteren Zeitraum verstreut. Insgesamt zählte das Reichsgesetzblatt 1918, also bis zum Ende der Monarchie, 390 Verlautbarungen, 48 Prozent davon waren Regierungsverordnungen nach dem KwEG. In der Regel bezogen sich die erlassenen Regierungsverordnungen explizit auf das KwEG als Rechtsgrundlage. Lediglich in Einzelfällen wurde die Rechtsgrundlage nicht angegeben, so dass nach Außen hin die Verordnung als Regie-

\footnotetext{
${ }^{80}$ MerKL, Verfassung 10, Anm. 1.

${ }^{81}$ Für die Untersuchung wurde primär das Reichsgesetzblatt und ergänzend die Stenographischen Protokolle des Abgeordnetenhauses herangezogen. Nicht berücksichtigt wurden Kundmachungen, die auf dem KwEG basierten.
}

rungsverordnung nach dem KwEG nicht erkennbar war. ${ }^{82}$ Warum in diesen Einzelfällen die Berufung auf das KwEG unterblieb, ist unklar handelte es sich schließlich in diesen Fällen oft um Rechtsakte des Amtes für Volksernährung bzw. des Handelsministers, die beide zu den häufigsten Anwendern des KwEG gehörten. Häufiger wurde in der Ersten Republik das KwEG als Rechtsgrundlage verschwiegen und lediglich auf „besondere Ermächtigungen des Staatsrates" verwiesen. ${ }^{83}$ Dass es sich dabei zum Teil trotzdem um Regierungsverordnungen nach dem KwEG handelte, ergibt sich lediglich aus deren Vorlage als solche im Parlament. ${ }^{84}$

Von den 304 Regierungsverordnungen nach dem KwEG, die in der Monarchie erlassen wurden, fielen 109 in den Zuständigkeitsbereich des Amtes für Volksernährung, 111 wurden vom Handelsminister, 21 vom Finanzminister, 19 vom Ackerbauminister, 10 vom Justizminister, 5 vom Verteidigungsminister, 5 vom Sozialminister, 4 vom Minister für Öffentliche Arbeiten und ebenfalls 4 vom Innenminister erlassen. ${ }^{85}$ Lediglich 16 Regierungsverordnungen nach dem KwEG wurden in der Monarchie vom Gesamtministerium erlassen. Von diesen 16 KwEGVerordnungen behandelte die Mehrzahl juristische Fragen, wie das Mietrecht ${ }^{86}$ oder die Stundung von Geldforderungen ${ }^{87}$ - aber auch die Einführung der Sommerzeit erfolgte 1918 mittels KwEG-Verordnung des Gesamtministeriums. ${ }^{88}$

\footnotetext{
${ }^{2}$ RGBl. 372/1917, RGBl. 479/1917, RGBl. 110/1918, RGBl. 204/1918, RGB1. 245/1918, RGB1. 309/1918.

${ }^{83}$ So bspw. StGBl. 29/1918, StGBl. 33/1918, StGBl. 50/1918, StGB1. 86/1918.

${ }^{84}$ Sten Prot KNV 1919, 21-24.

${ }^{85}$ In der Regel erfolgte die Erlassung einer Regierungsverordnung durch mehrere Regierungsmitglieder. In der durchgeführten Untersuchung für die Jahre 1917 und 1918 wurde für die Auswertung der Daten jeweils der erstgenannte Minister als primär zuständig gewertet.

${ }^{86}$ RGBl. 323/1917.

${ }^{87}$ RGB1. 495/1917, RGBl. 225/1918.

${ }^{88}$ RGBl. 87/1918, RGBl. 108/1918.
} 
Während 1917 die relative Mehrheit der KwEGVerordnungen in den Bereich des Handelsministeriums erlassen wurde (46) und das Amt für Volksernährung den zweiten Platz belegte (42), war die Situation 1918 genau umgekehrt: 67 KwEG-Verordnungen waren dem Amt für Volksernährung zurechenbar, 65 hingegen dem Handelsministerium.

Inhaltlich beschäftigten sich ein Viertel der Regierungsverordnungen nach dem KwEG mit dem Verkehr und Transport von Waren. Dabei kamen in der Regel Transportbescheinigungen zur Anwendung. Ein Siebentel der KwEGVerordnungen beschäftigte sich mit den Preisen der Waren, insbesondere für Obst und Gemüse wurden Höchstpreise festgesetzt. Auch die Bildung von Kriegsverbänden bzw. von Wirtschaftsverbänden erfolgte durch Regierungsverordnungen. $\mathrm{Zu}$ weiteren Materien, die mittels KwEG-Verordnungen erledigt wurden, zählten die Herstellung und der Verkehr mit Alkohol, die Bildung von Fach- und Wirtschaftsausschüssen, sowie die Beschlagnahme verschiedener für die Kriegsführung notwendiger Materialien. Beispielsweise mussten Gegenstände aus Kupfer und Kupferlegierungen abgeliefert werden, ${ }^{89}$ ebenfalls Orgelpfeifen wurden vom Ministerium für Landesvereidigung beansprucht. ${ }^{90}$

\section{KwEG in der Ersten Republik ${ }^{91}$}

\section{1. Überleitung des KwEG}

Das KwEG blieb - den staatrechtlichen Umbrüchen trotzend - bis zu seiner Aufhebung 1946 im Bestand der österreichischen Gesetze.

Die erste Überleitung erfolgte 1918 von der Monarchie zur Republik Deutschösterreich. Juris-

\footnotetext{
${ }^{89}$ RGB1. 403/1917.

${ }^{90}$ RGB1. 417/1917.

${ }^{91} \mathrm{Vgl}$. dazu den Beitrag von Ilse ReITER-ZatLouKAL in diesem Band.
}

tisch vollzog sich die revolutionäre Bildung des Staates Deutschösterreich im Herbst 1918. Mit $\S 16$ Staatsgründungsbeschluss ${ }^{92}$ erfolgte eine pauschale Rechtsüberleitung von der Monarchie zur Republik im Sinne einer materiellen Kontinuität bei formeller Diskontinuität. Lediglich einzelne Organe und Gesetze wurden durch den Staatsgründungsbeschluss abgeändert. Als gewaltenverbindendes Regierungs- und Gesetzgebungsorgan formierte sich bereits zuvor die Provisorische Nationalversammlung, die die Aufgaben des Reichsrates übernahm, ohne jedoch seine Nachfolgerin de lege zu werden. Das Herrenhaus und die Landtage wurden abgeschafft. ${ }^{93}$ Durch diese pauschale Rezeption kam auch das KwEG 1917 in den Rechtsbestand der jungen Republik. Angesichts der neuen Strukturen stellte sich bei der Anwendung des KwEG eine Vielzahl an Fragen: Angefangen von der Begriffsdefinition für Regierung, ${ }^{94}$ über den zeitlichen Anwendungsbereich ${ }^{95}$ bis hin zur Frage der Vorlagepflicht. ${ }^{96}$ Unsicherheit bestand allein schon hinsichtlich der rechtswirksamen Übernahme des KwEG in die Republik. Einige dieser Fragen klärte das Verfassungs-Übergangsgesetz 1920.

Das Verfassungs-Übergangsgesetz 1920 verfolgte das Ziel den Übergang in die neue Verfassung, das B-VG 1920, vorzubereiten und zu erleichtern. Zu diesem Zweck wurden Rechtsnormen des vorbundesstaatlichen Österreichs für einen Übergangszeitraum in Geltung belassen, so auch das KwEG 1917. Im von Hans Kelsen, Adolf Merkl und Georg Froehlich verfassten Kommentar heißt es zu dieser Gesetzesstelle,

\footnotetext{
92 Beschluß der Provisorischen Nationalversammlung für Deutschösterreich vom 30. 10. 1918, StGBl. 1/1918, über die grundlegenden Einrichtungen der Staatsgewalt.

${ }^{93}$ Art. 8 Gesetz v. 12. 11. 1918, StGBl. 5/1918, über die Staats- und Regierungsform von Deutschösterreich.

${ }_{94}$ Vgl. dazu Punkt 3.1.

${ }^{95}$ Vgl. dazu Punkt 3.2.

${ }^{96}$ Näher unter Punkt 2.3.
} 
dass dadurch „das bisher verfassungsrechtlich bedenkliche kriegswirtschaftliche Ermächtigungsgesetz vom Standpunkt der neuen Bundesverfassung aus legitimiert" ${ }^{\prime \prime 97}$ wurde. Eigens normiert wurde im $\S 17$ Abs. 2 VerfassungsÜbergangsgesetz, dass mittels Bundesgesetz festzustellen ist, wann die „durch die kriegerischen Ereignisse der Jahre 1914 bis 1918 hervorgerufenen außerordentlichen Verhältnisse“ "als behoben anzusehen sind“. .8

\subsection{KwEG-Verordnungen in der Praxis}

In den Anfangsjahren der Ersten Republik blieb die Anzahl der KwEG-Verordnungen, nun unter der Bezeichnung Vollzugsanweisungen, 99 hoch. Im Jahr 1919 wurden 206 KwEG-Verordnungen kundgemacht. ${ }^{100}$ Damit machten sie rund ein Drittel der im Staatsgesetzblatt kundgemachten Rechtsakte aus - 1919 verzeichnete das Staatsgesetzblatt insgesamt 611 Verlautbarungen. Die meisten KwEG-Verordnungen entfielen auf das Staatsamt für Handel und Gewerbe, Industrie und Bauten mit 76 Verordnungen. Den zweiten Platz belegte das Staatsamt für Volksernährung mit 42 Verordnungen nach dem KwEG, auffallend ist dabei der - im Vergleich zu den früheren Jahren - starke Rückgang der KwEGVerordnungen in diesem Bereich. In den folgenden Jahren wurden immer weniger KwEGVerordnungen erlassen, bis es sich schließlich nur noch um Einzelfälle handelte. Parallel zu dieser Entwicklung kamen im Rahmen der Gen-

\footnotetext{
${ }^{97}$ Kelsen, Froehlich, MerKL, Bundesverfassung 311.

98 § 17 Verfassungs-Übergangsgesetz 1920.

${ }^{99}$ Vgl. dazu HASIBA, Kriegswirtschaftliches Ermächtigungsgesetz 557, Anm. 92. Zur Begrifflichkeit vgl. MERKL, Republik Deutschösterreich 104-106.

${ }^{100}$ Die Berechnung erfolgte anhand der Stenographischen Protokolle der Konstituierenden Nationalversammlung. Hasiba gibt - ebenfalls mit Berufung auf die Stenographischen Protokolle - andere Zahlen an, die jedoch der Überprüfung nicht standhalten. HASIBA, Kriegswirtschaftliches Ermächtigungsgesetz 559.
}

fer Protokolle 1922 neue Wege der außerordentlichen Gesetzgebung hinzu. ${ }^{101}$

Mehrere Versuche das KwEG zu vernichten scheiterten. Bereits im Mai 1919 gab es einen Antrag auf Aufhebung des KwEG, 1928 und 1930 folgten weitere.102 Sie alle blieben unbehandelt, erfolglos. Sozialdemokratische Pläne, das KwEG mit der B-VG Novelle 1929 zu beseitigen, scheiterten. Zwar sah die Bundesverfassung nun ein Notverordnungsrecht des Bundespräsidenten vor, ${ }^{103}$ doch wurde im Verfassungsübergangsgesetz das KwEG nach wie vor rezipiert.

Kritische Stimmen bezüglich der KwEG-Praxis fanden sich nicht nur in der Politik. Als im November 1922 eine Bestimmung des Allgemeinen bürgerlichen Gesetzbuches mit Hilfe des KwEG abgeändert wurde, rief dieser Schritt Entrüstung in den Juristenkreisen hervor. Zwar wurde die neue Bestimmung inhaltlich nicht beanstandet, doch richtete sich die Kritik gegen die Heranziehung des KwEG. ${ }^{104}$

Unterschiedlich wurde die Vorlagepflicht in der Ersten Republik gehandhabt. Während zunächst die KwEG-Verordnungen dem parlamentarischen Körper vorgelegt wurden, unterblieb diese Praxis in den späten 1920er Jahren. ${ }^{105}$ Organisatorisch lag die Zusammenstellung der entsprechenden Verordnungen in den Händen der Staatskanzlei bzw. später des Bundeskanzleramtes. Mit diesen Agenden beschäftigten sich in der Staatskanzlei - wie die Akten des Archivs der Republik bezeugen - durchaus namhafte Juristen wie der nachmalige Verfassungsrichter

${ }^{101}$ Vgl. dazu den Beitrag von Gerald KOHL in diesem Band.

102 HASIBA, Kriegswirtschaftliches Ermächtigungsgesetz 558, 561, 563.

${ }^{103} \mathrm{Vgl}$. dazu den Beitrag von Ewald WIEDERIN in diesem Band.

104 Vgl. dazu ausführlicher STAUDIGL-CIECHOWICZ, Zivilrecht 54-57.

${ }^{105}$ HASIBA, Kriegswirtschaftliches Ermächtigungsgesetz 561 . 
Georg Froehlich und der Staatsrechtler Adolf Merkl.106

\subsection{KwEG in der Rechtsprechung des VfGH}

Das Kriegswirtschaftliche Ermächtigungsgesetz wurde des Öfteren - vor allem bis 1922 - bei der Rechtsprechung des Verfassungsgerichtshofes berücksichtigt. Zwar beschäftigen sich nur wenige Fälle direkt mit dem KwEG, ${ }^{107}$ doch lag das KwEG mehreren überprüften Bestimmungen zu Grunde und wurde folglich in den Entscheidungen des VfGH erwähnt.108 Ab 1923 gibt es immer weniger Erwähnungen des KwEG, ab 1929 findet sich das KwEG gar nicht mehr im Register der in den Entscheidungen zitierten Gesetze. ${ }^{109}$ Selbst aus den Erkenntnissen des Verfassungsgerichtshofes, die das KwEG lediglich beiläufig zitierten, lassen sich Schlüsse für dessen Anwendbarkeit und Anwendung ziehen

106 ÖStA, AdR, BKA Stk, Bka alt ZR186.

${ }^{107}$ Erk. v. 24. 1. 1921, Zl. D 2/20; Erk. v. 12. 5. 1921, Zl.

D 1/21; Erk. v. 15. 12. 1921, Zl. D 7/21; Erk. v. 13.3. 1922, Zl. V 2/22; Erk. v. 21. 6. 1922, Zl. V 6/22; Erk. v. 11. 12. 1922, Zl. V 19/22; Erk. v. 15. 12. 1922, Zl. V 22/22; Erk. v. 21. 12. 1922, Zl. B 78/22; Erk. v. 23. 6. 1924, Zl. V 3/24; Erk. v. 15. 12. 1924, Zl. V 6/24; Erk. v. 5. 7. $1927, \mathrm{Zl}$. V 2/27.

108 1919: Erk. v. 16. 12. 1919, Zl. 331; Erk. v. 16. 12. 1919, Zl. 332; Erk. v. 17. 12. 1919, Zl. 333; Erk. v. 18. 12. 1919, Z1. 335.

1920: Erk. v. 24. 2. 1920, Zl. 12; Erk. v. 24. 2. 1920, Zl. 14; Erk. v. 25. 2. 1920, Zl. 15; Erk. v. 25. 2. 1920, Zl. 17; Erk. v. 8. 6. 1920, Zl. 34; Erk. v. 9. 6. 1920, Zl. 35; Erk. v. 10. 6. 1920, Zl. B. 9/20; Erk. v. 12. 10. 1920, Zl. B 12/20; Erk. v. 14. 10. 1920, Zl. B 16/20; Erk. v. 14. 10. 1920, Zl. B 17/20; Erk. v. 15. 10. 1920, Zl. A 16/20.

1921: Erk. v. 29. 4. 1921, Zl. B 25/20; Erk. v. 30. 4. 1921, Zl. 29/20; Erk. v. 16. 12. 1921, Zl. B 45/21; Erk. v. 17. 12. 1921, Zl. B 35/21.

1922: Erk. v. 14. 3.1922, Zl. A 65/21; Erk. v. 16. 3. 1922, Zl. B 3/22; Erk. v. 11. 10. 1922, Zl. A 36/22; Erk. v. 12. 12. 1922, Zl. B 81/22.

1923: Erk. v. 19. 6. 1923, Zl. V 5/23.

1928: Erk. v. 11. 12. 1928, Zl. G 5/28.

${ }^{109}$ Kein entsprechendes Register gibt es für die Jahre 1932-1933.
- so beispielsweise den ermächtigten Personenkreis $^{110}$ oder seine sanierende Wirkung ${ }^{111}$. Besonders häufig fand das KwEG in den Entscheidungen des VfGH zur Einschränkung des Grundrechts des freien Aufenthaltes gem. Art 6 StGG über die allgemeinen Rechte der Staatsbürger Erwähnung. ${ }^{112}$ Dabei sah der VfGH Einschränkungen des Grundrechts des freien Aufenthaltes basierend auf dem KwEG als zulässig an. ${ }^{113}$

\section{KwEG im Austrofaschismus ${ }^{114}$}

Dem KwEG wird eine tragende Rolle beim Untergang der Ersten Republik zugeschrieben. ${ }^{115}$ 1946 erklärte der SPÖ-Abgeordnete Eduard Weikhart anlässlich der Aufhebung des KwEG, dass „das kriegswirtschaftliche Ermächtigungsgesetz mit ein Anlaß dazu war, daß Hitler im März 1938 ein nicht mehr demokratisches und darum doppelt wehrloses Österreich widerstandslos besetzen konnte. "116 Wesentliche Bestimmungen, die einen funktionierenden Rechtsstaat garantierten, wurden durch KwEGVerordnungen ab 1933 aufgehoben bzw. abgeändert - bspw. die Pressefreiheit und die öffentliche Gerichtsbarkeit. ${ }^{117}$ Schließlich erfolgte sogar die Erlassung der Verfassung 1934 mittels KwEG-Verordnung. ${ }^{118}$ Mit 1934 hatte das KwEG ausgedient, durch das neue vom sogenannten Rumpfparlament beschlossene Ermächtigungs-

\footnotetext{
110 Siehe oben Punkt 3.1.

111 Siehe oben Punkt 2.3.

112 Vgl. dazu den Beitrag von Ilse ReITER-ZATLOUKAL in diesem Band.

${ }^{113}$ Erk. v. 25. 2. 1920, Zl. 15.

${ }^{114}$ Vgl. zu diesem Thema den Beitrag von Martin F. POLASCHEK in diesem Band.

115 Vgl. ausführlich HUEMER, Sektionschef Hecht

116 Sten Prot NR V. GP, 688.

117 Vgl. dazu HELLER, Verfassungsgerichtshof.

118 Vgl. zur Verfassung 1934 MERKL, Verfassung.
} 
gesetz $1934^{119}$ die Regierung noch umfangreichere Vollmachten bekommen. ${ }^{120}$

Nach dem Inkrafttreten der Maiverfassung 1934 war es strittig, ob das KwEG weiterhin galt oder nicht. Eine explizite formelle Aufhebung des Gesetzes erfolgte bis zum „Anschluss“ 1938 nicht. ${ }^{121}$

\section{KwEG in der zeitgenössischen juristischen Literatur ${ }^{122}$}

In der zeitgenössischen juristischen Berichterstattung spielte das KwEG erst spät eine zentralere Rolle. Unmittelbar nach der Erlassung des Gesetzes wurde es nur vereinzelt literarisch behandelt, so vom Wiener Staatsrechtler Leo Wittmayer. ${ }^{123}$ Wittmayer spezialisierte sich auf rechtsvergleichende Aspekte des österreichischen und deutschen Verfassungsrechts und beleuchtete unter diesem Blickwinkel 1917/18 die Notverordnungsrechte beider Staaten. ${ }^{124}$ Für Wittmayer hatten KwEG-Verordnungen einen geringeren Stellenwert als Notverordnungen, er verneinte ihre vermeintliche gesetzesändernde Wirkung. ${ }^{125}$ Erst mit der Zuspitzung der politischen Lage in den frühen 1930er Jahren rückte das KwEG in den Mittelpunkt juristischer Untersuchungen. Mehrere Abhandlungen beleuchten das KwEG im Zusammenhang mit bestimmten KwEG-Verordnungen der 1930er Jahre ${ }^{126}$

\footnotetext{
${ }^{119}$ BGBl. I 255/1934.

${ }^{120} \mathrm{Vgl}$. dazu den Beitrag von Helmut WOHNOUT in diesem Band.

${ }^{121}$ Erläuternde Bemerkungen, 153 Blg V. GP.

122 Vgl. dazu auch HUEMER, Sektionschef Hecht 150156.

${ }^{123}$ Vgl. zu Wittmayer OLECHOWSKI, EHS, STAUDIGLCiECHOWICZ, Rechts- und Staatswissenschaftliche Fakultät 472f.

${ }^{124}$ WITTMAYER, Ermächtigungsgesetz.

125 Vgl. dazu oben Punkt 3.5.

${ }^{126}$ BERGER, Ustawodawstwo; SUEß, Verordnung; LAYER, Ermächtigungsbereich; HORN, Gesetzgeber.
}

oder der Erlassung der Verfassung 1934. ${ }^{127}$ Dabei hatten die Autoren regierungskritischer Beiträge sogar mit Repressalien zu rechnen. So wurde der Staatsrechtler Max Layer vorzeitig in den Ruhestand versetzt, ${ }^{128}$ nachdem er 1933 einen sehr kritischen Artikel zur Anwendung des kriegswirtschaftlichen Ermächtigungsgesetzes in der gleichgeschalteten reichsdeutschen Zeitschrift "Verwaltungsrecht" publizierte.129 Layer prangerte die KwEG-Praxis der autoritären Regierung an und verdeutlichte anhand mehrerer Beispiele seine „schwere[n] verfassungsrechtliche[n] Bedenken“ gegen viele Regierungsverordnungen ${ }^{130}$, so bspw. der Einschränkung der Pressefreiheit.131 Zur „bedenklichste[n] der verfassungswidrigen Verordnungen“ erklärte er jedoch die KwEG-Verordnung, mit der die Organisation des VfGH abgeändert und dieser folglich ausgeschaltet wurde. ${ }^{132}$ Ebenfalls einige andere Wiener Rechtsgelehrte behandelten im gleichen Zeitschriftenband wie Layer die rezenten staatsrechtlichen Entwicklungen kritisch. $\mathrm{Zu}$ ihnen gehörte auch Adolf Merkl, der darüber hinaus mehrere Artikel zum KwEG und der verfassungsrechtlichen Situation verfasste und in juristischen Zeitschriften sowie der Tagespresse publizierte. Dabei ging er der Frage nach, inwiefern das 1929 eingeführte Notverordnungsrecht des Bundespräsidenten mit

\footnotetext{
${ }^{127}$ GÜRKE, Verfassung; RASCHHOFER, Verfassung; MERKL, Verfassung.

${ }^{128}$ Layer hatte zwar das Mindestalter für den Ruhestand schon erreicht, allerdings war es zuvor unüblich Professoren in diesem Alter auch tatsächlich in den Ruhestand zu schicken. Vgl. dazu STAUDIGLCIECHOWICZ, Dienst-, Habilitations- und Disziplinarrecht 203-213.

${ }^{129}$ Vgl. ausführlich dazu OLECHOWSKI, EHS, STAUDIGLCIECHOWICZ, Rechts- und Staatswissenschaftliche Fakultät 505-508; OLECHOWSKI, STAUDIGL-CIECHOWICZ, Staatsrechtslehre 234-239.

${ }^{130}$ LAYER, Ermächtigungsbereich 208.

${ }^{131}$ BGBl. 41/1933.

${ }^{132}$ LAYER, Ermächtigungsbereich 212.
} 
dem KwEG zusammenspielte, ${ }^{133}$ er kritisierte die Verfassungswidrigkeit der Suspension der Pressefreiheit mittels Regierungsverordnung, 134 sowie allgemein den Missbrauch des KwEG. ${ }^{135}$

Noch vor der Ausschaltung des Nationalrates beschäftigte sich der Rechtsanwalt Emil Sueß mit dem KwEG im Zusammenhang mit der Regierungsverordnung über die Geltendmachung der Haftungen nach dem Zusammenbruch der "Creditanstalt". ${ }^{136}$ Sueß sah die Regierungsverordnung als nicht durch das KwEG gedeckt und begründete dies mit der Verletzung des sachlichen Ermächtigungsbereiches. Seiner Ansicht nach reichte nicht jeder beliebige wirtschaftliche Anknüpfungspunkt, vielmehr war ein Zusammenhang mit der Kriegswirtschaft bzw. den Kriegsfolgen notwendig und einen solchen verneinte er in diesem Fall. Seine warnenden Worte muten fast prophetisch an: „Wenn selbst zu anderer Zeit und aus anderem Anlasse derartige Verordnungen zulässig wären, könnte mit der fadenscheinigen Begründung wirtschaftlicher Gefährdung jederzeit das Parlament ausgeschaltet, und die ganze, in den Gesetzen niedergelegte Lebensarbeit der hervorragendsten Juristen durch eine Justizministerialverordnung nach beliebigem Maße außer Kraft gesetzt werden. Die Rechtsprechung der Geschworenen und anderer Gerichte kann im Einzelfalle und in größerem Umfange auch wirtschaftliche Interessen gefährden und dasselbe kann auch von vielen bewährten Bestimmungen des materiellen und formellen Rechtes gesagt, und diese können dann zeitweise oder für einzelne Personen auch außer Kraft gesetzt werden."137 Auch in der ausländischen juristi-

\footnotetext{
${ }^{133}$ MERKL, Verordnungsvollmacht. Vgl. dazu oben 3.3. ${ }^{134}$ MerKL, Pressefreiheit.

${ }^{135}$ MERKL, Verfassungskampf.

${ }^{136}$ SUEß, Verordnung. Es handelt sich dabei um BGBl. 303/1932, vgl. dazu näheres im Beitrag von Ilse REITER-ZATLOUKAL in diesem Band.

137 SUEß, Verordnung 231.
}

schen Literatur fehlten Beiträge zum KwEG und dessen Anwendung in den 1930er Jahren nicht. Während Adolf Berger sich in einer polnischen Zeitschrift mit den Regierungsverordnungen zur Bekämpfung staatsfeindlicher Tätigkeit auseinandersetzte und dabei das KwEG kurz streifte, ${ }^{138}$ fasste Norbert Gürke in der deutschen Zeitschrift "Archiv des öffentlichen Rechts" die neue österreichische Verfassung 1934 zusammen. ${ }^{139}$ Gürke, wie auch Hermann Raschhofer, der eine kurze Darstellung der Verfassung 1934 in der deutschen Zeitschrift für ausländisches öffentliches Recht und Völkerrecht publizierte, ${ }^{140}$ kritisierten die KwEG-Praxis der austrofaschistischen Regierung auf das Heftigste.

\section{KwEG nach dem 2. Weltkrieg ${ }^{141}$}

Österreich deklarierte bereits in der Unabhängigkeitserklärung die Rückkehr zum B-VG. Während diese 1945 noch an das B-VG 1920 anknüpfte, $^{142}$ erklärte das Verfassungs-Überleitungsgesetz 1945 die Rückkehr zum B-VG in der Fassung von 1929, für andere verfassungsrechtliche Bestimmungen galt der 5. März 1933 als Stichtag. ${ }^{143}$ Durch diesen Automatismus wurde folglich auch das KwEG in die 2. Republik übergeleitet.

Während die parlamentarische Arbeit nach dem Ende des 2. Weltkrieges zunächst faktisch undurchführbar war, regierte bis zur Nationalratswahl Ende November 1945 die Provisorische Staatsregierung als gewaltenverbindendes Organ gemäß der Vorläufigen Verfassung. Die österreichische Gesetzgebung unterlag der Kon-

\footnotetext{
138 BERGER, Ustawodawstwo.

${ }^{139}$ GÜRKE, Verfassung.

${ }^{140}$ RASCHHOFER, Verfassung.

${ }^{141} \mathrm{Vgl}$. dazu auch grundlegend HASIBA, Kriegswirtschaftliches Ermächtigungsgesetz 543-545.

${ }^{142}$ Art. 1 Unabhängigkeitserklärung, StGB1. 1/1945.

${ }^{143}$ Art. 1 Verfassungs-Überleitungsgesetz, StGBl. 4/1945.
} 
trolle der Alliierten. Während nach dem 1. Alliierten Kontrollabkommen alle Gesetze einer Zustimmung des Alliierten Rates bedurften, erfolgte durch das 2. Alliierten Kontrollabkommen Ende Juni 1946 eine Lockerung dieser Bestimmung. Lediglich Verfassungsgesetze benötigten nach wie vor eine schriftliche Genehmigung, bei allen anderen Gesetzen wurde die Genehmigung vermutet, so nicht innerhalb von 31 Tagen ein Einspruch erfolgte. ${ }^{144}$

In der Eröffnungssitzung des neugewählten Nationalrates Mitte Dezember 1945 im Sitzungssaal des ehemaligen Abgeordnetenhauses ${ }^{145}$ kam das KwEG zur Sprache. ${ }^{146}$ Der Berichterstatter, ÖVP-Abgeordneter Felix Hurdes, stellte das als Regierungsvorlage eingebrachte VerfassungsÜbergangsgesetz $1945^{147}$ vor. Entsprechend der von der Provisorischen Staatsregierung gemachten Proklamation wurden dem Nationalrat Übergangsvorschriften vorgelegt, die die Rückkehr zum B-VG 1929 ermöglichen sollten. Ziel war es "das klaglose Funktionieren des verfassungs- und verwaltungsmäßigen Lebens in Österreich auf allen Gebieten “148 zu ermöglichen und das B-VG in der Fassung von 1929 mit einzelnen Änderungen in Geltung zu setzen. Des Weiteren sah die Regierungsvorlage die Weitergeltung der Verfassungs-Übergangsgesetze aus 1920 und 1929 vor, allerdings mit gewissen Einschränkungen. So hießt es in den Erläuternden Bemerkungen: „hervorzuheben [wäre], daß [...] das sogenannte kriegswirtschaftliche Ermächtigungsgesetz in den Katalog der weitergeltenden Vorschriften nicht wieder aufgenommen wurde in der Annahme, daß im Hinblick auf die politische Geschichte dieser Verordnungsermächti-

\footnotetext{
${ }^{144}$ OLECHOWSKI, Rechtsgeschichte 118-120.

145 Schicksalswahl 34.

${ }^{146}$ Sten Prot NR V. GP, 12; HASIBA, Kriegswirtschaftliches Ermächtigungsgesetz 543.

${ }^{147} \mathrm{RV}, 1$ Blg NR V. GP.

${ }^{148}$ Erläuternde Bemerkungen, 1 Blg NR V. GP, 4.
}

gung von ihrer weiteren Handhabung abgesehen werden sollte. “149

Die Bedeutung der Aufhebung des kriegswirtschaftlichen Ermächtigungsgesetzes für die "neue Demokratie“ betonte Hurdes bei der Berichterstattung. Er sah darin, „daß man sich von vornherein auf den Standpunkt stellt, auf dieses kriegswirtschaftliche Ermächtigungsgesetz nie mehr zurückzugreifen", einen Beweis "für die Stärke der neuen Demokratie“ und erntete für seine Worte lebhaften „Beifall und Händeklatschen“.150 Der Entwurf des VerfassungsÜbergangsgesetzes 1945 wurde in zweiter und dritter Lesung einstimmig angenommen. ${ }^{151} \mathrm{Am}$ gleichen Tag stimmte der Bundesrat über den Gesetzesbeschluss des Nationalrates ab und fasste den Beschluss, keinen Einspruch zu erheben. ${ }^{152}$

Trotz dieser übereinstimmenden Beschlüsse wurde das Verfassungs-Übergangsgesetz 1945 nicht kundgemacht, es scheiterte an der $\mathrm{Zu}$ stimmung des Alliierten Rates. ${ }^{153}$ Ende März 1946 teilte dieser dem Bundeskanzler mit, dass der Alliierten Rat bis 1. Juli 1946 die Vorlage „einer definitiven Verfassung, die sich auf demokratischen Prinzipien gründet" verlange. Dem Verfassungs-Übergangsgesetz 1945 hingegen wurde die Zustimmung verweigert, da „eines der Elemente der Meinung [war], daß dieser Gesetzentwurf [sic] nicht der neuen Lage entspricht, in der sich der österreichische Staat befindet. Die anderen drei Elemente waren, vorbehaltlich gewisser Abänderungen und in Erwartung einer ständigen Verfassung, bereit, dem Entwurf dieses provisorischen Gesetzes ihre

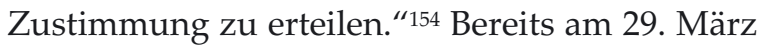

\footnotetext{
${ }^{149}$ Erläuternde Bemerkungen, 1 Blg NR V. GP, 4.

150 Sten Prot NR V. GP, 12.

${ }^{151}$ Sten Prot NR V. GP, 13.

152 Sten Prot BR v. 19. 12. 1945, 5.

${ }^{153}$ Sten Prot NR V. GP, 69f. HASIBA, Kriegswirtschaftliches Ermächtigungsgesetz 543f; HeLLER, Verfassungsgerichtshof 310.

154 Sten Prot NR V. GP, 69.
} 
1946 berichtete die Wiener Zeitung von der Weigerung der Sowjetunion die „veraltete Verfassung" auch nur als Provisorium zu akzeptieren. ${ }^{155}$ Freilich richteten sich die Beanstandungen nicht gegen die Aufhebung des KwEG, trotzdem blieb es mangels des Inkrafttretens des Verfassungs-Übergangsgesetzes 1945 weiterhin in Kraft.

Den Wünschen des Alliierten Rates wurde in weiterer Folge nicht entsprochen, sondern die Geltung des B-VG 1929 aus dem VerfassungsÜberleitungsgesetz vom Mai 1945, das der Alliierten Rat genehmigt hatte, hergeleitet. ${ }^{156}$ Das löste jedoch nicht das Problem der Weitergeltung des KwEG. Anfang Juli 1946 langte als Regierungsvorlage im Nationalrat das Bundesverfassungsgesetz, womit das KwEG aufgehoben wird, ein. ${ }^{157}$ Angesichts der Tatsache, dass die Geltung des KwEG nach dem Inkrafttreten der Maiverfassung 1934 strittig war, wollte man nun mit einer formellen Aufhebung des Gesetzes auf Nummer sicher gehen, um „bei der bestehenden Rechtskontinuität für die Zukunft jede Möglichkeit für eine wenn auch nur eingeschränkte Handhabung dieses Gesetzes zu nehmen". ${ }^{158}$ Bereits Mitte Juli lag der Bericht des Verfassungsausschusses vor. Dieser begrüßte die Regierungsvorlage und bekräftigte, es sei an der Zeit, mit der formellen Aufhebung des KwEG „endlich Ordnung zu schaffen“.159 Auch wurde die Notwendigkeit des KwEG bestritten, für "staatliche Notstände“ sah der Verfassungsausschuss die im Art 18 B-VG verankerten Notrechte des Bundespräsidenten als ausreichend an.

Am 25. Juli 1946 wurde der Ausschussbericht im Plenum besprochen. Auffallend ist, dass sich

\footnotetext{
155 Wiener Zeitung v. 29. 3. 1946, Nr. 75, S. 1.

156 Schicksalswahl 35; HeLLER, Verfassungsgerichtshof 311.

157 RV, 153 Blg V. GP.

${ }^{158}$ Erläuternde Bemerkungen, 153 Blg V. GP.

${ }^{159}$ AB, 181 Blg V. GP.
}

abgesehen von Eduard Ludwig, ÖVP Abgeordneter und Berichterstatter, nur Mitglieder der SPÖ und KPÖ in der Debatte zu Wort meldeten - das KwEG war nach wie vor politisch ein sehr unbequemes Thema.

Der SPÖ-Abgeordnete Eduard Weikhart gedachte kurz der unrühmlichen Vergangenheit des KwEG und richtete dabei seine Rede insbesondere gegen die ÖVP als er feststellte, dass „das kriegswirtschaftliche Ermächtigungsgesetz [...] dem Faschismus in Österreich Tür und Tor geöffnet [hat], und viele Herren, die es damals mit Freude gutgeheißen haben (starke Unruhe und Zwischenrufe von seiten [sic] der Österreichischen Volkspartei) oder aber auch zu feige waren, dagegen aufzutreten, [...] heute wieder im öffentlichen Leben [stehen] und [...] so [tun], als ob sie stets die Demokratie mit dem großen Löffel gegessen hätten“.160 Der KPÖAbgeordnete Johann Koplenig sah in der Aufhebung des KwEG einen „Schritt zur Demokratisierung", er plädierte, einen Schritt weiter zu gehen und Österreich eine neue Verfassung zu geben. ${ }^{161}$

Den Abschluss machte die SPÖ-Abgeordnete Paula Wallisch mit den Worten „Wir beseitigen heute dieses kriegswirtschaftliche Ermächtigungsgesetz. Es ist wie in einer Gerichtssitzung: die Schuldigen hat die Geschichte schuldig gesprochen - das Mordinstrument wird hoffentlich für alle Zeiten weggeräumt!“162

Gerade Paula Wallischs Lebensweg nahm im Austrofaschismus eine tragische Wende. Ihr Gatte, Koloman Wallisch, war als Führungsperson in der Arbeiterbewegung nach dem Februaraufstand 1934 verhaftet, am Folgetag standrechtlich verurteilt und anschließend exekutiert worden. ${ }^{163}$ Zwar war die Todesstrafe im or-

\footnotetext{
160 Sten Prot NR V. GP, 687.

161 Sten Prot NR V. GP, 689.

162 Sten Prot NR V. GP, 691.

163 POLASCHEK, "ständisch-autoritär“ 252f. Vgl. auch NECK, Wallisch 459-462.
} 
dentlichen Verfahren 1919 abgeschafft worden, bestand aber nach wie vor im Verfahren vor dem Standgericht. Bezeichnend für die austrofaschistische KwEG-Praxis ist, dass gerade auch die Verhängung des Standrechts mittels einer Regierungsverordnung nach dem KwEG erleichtert wurde. ${ }^{164}$

Die Aufhebung des KwEG wurde einstimmig am 25. Juli 1946 im Nationalrat beschlossen. Mit der Kundmachung des Bundesverfassungsgesetzes am 10. September 1946 im Bundesgesetzblatt war auch das formelle Bestehen des Kriegswirtschaftlichen Ermächtigungsgesetzes beendet. ${ }^{165}$

\section{Korrespondenz:}

Dr. Kamila StAUdigl-CiechOWICZ, LL.M.

Universität Wien

Forschungsstelle für Rechtsquellenerschließung

Postgasse 7, Stiege 1

1010 Wien

kamila.staudigl-ciechowicz@univie.ac.at

ORCID Nr. 0000-0002-7018-535X

\section{Abkürzungen:}

Erk. Erkenntnis

KwEG Kriegswirtschaftliches Ermächtigungsgesetz, RGBl. 307/1917

KwEVO Kriegswirtschaftliche Ermächtigungsverordnung, RGB1. 274/1914

Siehe auch das allgemeine Abkürzungsverzeichnis: [http://www.rechtsgeschichte.at/files/abk.pdf]

\section{Literatur:}

Adolf BERGER, Ustawodawstwo austrjackie 1933-1934 przeciw terrorowi i działalności antypaństwowej, in: Przegląd prawa i administracji 60 (1935) 12-42.

Norbert GÜRKE, Die österreichische „Verfassung 1934“, in: Archiv des öffentlichen Rechts 25 NF (1934), 178-255.

Gernot D. HASIBA, Das Kriegswirtschaftliche Ermächtigungsgesetz (KWEG) von 1917. Seine Entstehung und seine Anwendung vor 1933, in: Rechtswiss. Fakultät der Univ. Salzburg (Hg.), Aus Österreichs Rechtsleben in Geschichte und Gegenwart. Festschrift für Ernst C. Hellbling zum 80. Geburtstag (Berlin 1981) 543-565.

DERS., Das Notverordnungsrecht in Österreich (18481917). Notwendigkeit und Mißbrauch eines "Staatserhaltenden Instrumentes" (= Studien zur Geschichte der österreichisch-ungarischen Monarchie 22, Wien 1985).

Kurt HeLLER, Der Verfassungsgerichtshof. Die Entwicklung der Verfassungsgerichtsbarkeit in Österreich von den Anfängen bis zur Gegenwart (Wien 2010).

Fritz HORN, Gesetzgeber, Gesetz und die kriegswirtschaftliche Ermächtigung, in: JBl. 63 (1934) 206-209, 232-236.

Peter HuEMER, Sektionschef Robert Hecht und die Zerstörung der Demokratie in Österreich. Eine historisch-politische Studie (Wien 1975).

Hans Kelsen, Georg Froehlich, Adolf Merkl (Hgg.), Die Bundesverfassung vom 1. Oktober 1920 (Wien-Leipzig 1922, ND: Wien 2003).

Max LAYER, Ermächtigungsbereich des kriegswirtschaftlichen Ermächtigungsgesetzes, in: Verwaltungsarchiv 38 (1933), 203-218.

Hannes LeIDINGER, Verena MoRITZ, Das Kriegswirtschaftliche Ermächtigungsgesetz (KWEG) vor dem Hintergrund der österreichischen Verfassungsentwicklung, in: Florian WENNINGER, Lucile DREIDEMY (Hgg.), Das Dollfuss/SchuschniggRegime 1933-1938. Vermessung eines Forschungsfeldes (Wien u.a. 2013) 449-470.

Adolf MERKL, Die Verordnungsgewalt im Kriege. Teil III: Kaiserliche und behördliche Verordnung, in: JB1. 45 (1916), 397-399.

DERS., Die Verfassung der Republik Deutschösterreich. Ein kritisch-systematischer Grundriß (Wien-Leipzig 1919).

DERS., Die Suspension der Pressefreiheit, in: Neue Freie Presse v. 9. 3. 1933, Nr. 24601, Abendblatt 2.

DERS., Der Verfassungskampf, in: Der österreichische Volkswirt 25 (1935) 609-612.
${ }^{164}$ BGBl. 501/1933.

165 BGBl. 143/1946. 
DERS., Die Frage der Geltung des kriegswirtschaftlichen Ermächtigungsgesetzes und seines Verhältnisses zur Verordnungsvollmacht des Bundespräsidenten, in: JBl. 62 (1933) 137-141.

DERS., Die ständisch-autoritäre Verfassung Österreichs. Ein kritisch-systematischer Grundriß (Wien 1935).

Rudolf NECK, Koloman Wallisch vor dem Standgericht, in: Gerhard PfERSCHY (Hg.), Siedlung Macht und Wirtschaft. Festschrift für Fritz Posch zum 70. Geburtstag (Graz 1981) 455-464.

Thomas OLECHOWSKI, Rechtsgeschichte. Einführung in die historischen Grundlagen des Rechts (Wien 2016).

Ders., Kamila StaUdigl-CiechoWICZ, Die Staatsrechtslehre an der Universität Wien 1933-1938, in: Ilse ReITER-ZATlOUKAL, Christine ROTHLÄNDER, Pia SCHÖLNBERGER (Hgg.), Österreich 1933-1938. Interdisziplinäre Annäherung an das Dollfuß/Schuschnigg-Regime (Wien u.a. 2012) 227-241.

DeRs., Tamara EHS, Kamila STAUDIGL-CiECHOWICZ, Die Wiener Rechts- und Staatswissenschaftliche Fakultät 1918-1938 (=Schriften des Archivs der Universität Wien 20, Göttingen 2014).

Martin POLASCHEK, Statt „ständisch-autoritär“ ständig autoritär. Die Steiermark zwischen 1933 und 1938, in: Alfred Ableitinger (Hg.), Bundesland und Reichsgau. Demokratie, "Ständestaat" und NSHerrschaft in der Steiermark 1918 bis 1945 (=Geschichte der Steiermark 9/I, Wien-KölnWeimar 2015) 239-287.
Hermann RASCHHOFER, Österreichs neue Verfassung 1934, in: Zeitschrift für ausländisches öffentliches Recht 4 (1934) 846-857.

Schicksalswahl 1945. Katalog zur Sonderausstellung im österreichischen Parlament aus Anlass des 60. Jahrestages der Nationalratswahl vom 25. November 1945 (Wien 2005).

Kamila Staudigl-Ciechowicz, Das Dienst-, Habilitations- und Disziplinarrecht der Universität Wien 1848-1938. Eine rechtshistorische Untersuchung zur Stellung des wissenschaftlichen Universitätspersonals (= Schriften des Archivs der Universität Wien Bd. 22, Göttingen 2017).

DIES., Österreich. Das österreichische Zivilrecht und die österreichische Zivilrechtswissenschaft in der Zwischenkriegszeit, in: Martin LÖHNIG, Stephan WAGNER (Hgg.), „Nichtgeborene Kinder des Liberalismus“? - Zivilgesetzgebung im Mitteleuropa der Zwischenkriegszeit“" (Tübingen 2018) 39-69.

Emil SUEß, Zur Verordnung des Bundesministers für Justiz vom 1. Oktober 1932 über die Geltendmachung der im 7. Credit-Anstaltsgesetz angeführten Haftungen, in: RZ 25 (1932) 230f.

Leo WitTMAYER, Ein Beitrag zum deutschen und österreichischen Notverordnungsrechte im Kriege, in: Archiv des öffentlichen Rechts 37 (1918) 35-55.

DERS., Das kriegswirtschaftliche Verordnungsrecht und das neue österreichische Ermächtigungsgesetz, in: Archiv des öffentlichen Rechts 37 (1918) 359-369. 